\title{
LETTER
}

\section{Early administration of corticosteroids and mortality}

\author{
Charles L Sprung* and Baruch M Batzofin \\ See related research by Park et al., http://ccforum.com/content/16/1/R3
}

We read the paper by Dr Park and colleagues [1] with interest. The evaluation of the association between the early administration of corticosteroids and mortality in patients with septic shock is extremely important. The authors demonstrated that the median time to steroid initiation was shorter in survivors and mortality rates increased with increasing quintiles of time until the initiation of steroids. The authors correctly note the limitations of their study given its retrospective design with the possibility of selection bias related to more severely ill patients receiving steroids later. As the baseline severity or number of organ failure at the time of steroid treatment was not different between the early and later treated patients and the adjusted multivariate analysis corrected for age, gender, time to initiation of antibiotics and illness severity (Simplified Acute Physiology
Score (SAPS) 3) still showed a significant association between the time to initiation of steroid therapy and 28-day mortality, the authors concluded that there is a significant association between early steroid initiation and decreased mortality in septic shock patients. Unfortunately, the data for illness severity (SAPS3 and Sequential Organ Failure Assessment (SOFA) scores) as assessed by quintiles over time was not reported. These data according to the authors during the review process showed significant increases of SAPS3 $(P<0.01)$ and SOFA $(P<0.05)$ scores over time, which could also explain the increased mortality in patients receiving corticosteroids later. Therefore, the findings of increased mortality in patients receiving steroids later could be related to the initiation time of the steroids or alternatively to their increased severity of illness.

\section{Authors' response}

Kyeongman Jeon, Hye Yun Park and Sookyoung Woo

We thank Drs Sprung and Batzofin for their thoughtful comments regarding our recent article [1]. We understand their concern that more severely ill patients might received delayed initiation of low-dose corticosteroid therapy compared to patients who received early therapy. However, the baseline severity of illness or number of organ failures was not different between early vs. late treatment groups. The 28-day mortality rates increased significantly with increased quintiles of time to initiation of low-dose corticosteroid therapy as described in the original article $(P=0.0107)$ [1]. Simplified Acute Physiology Score 3 (SAPS 3) and Sequential Organ Failure Assessment (SOFA) score by quintiles were significantly different when tested with Kruskal-Wallis test $(P=0.0492)$. However, because this test does not identify where the

*Correspondence: sprung@cc.huji.ac.il

Department of Anesthesiology and Critical Care Medicine, Hadassah Hebrew University Medical Center, Jerusalem, Israel

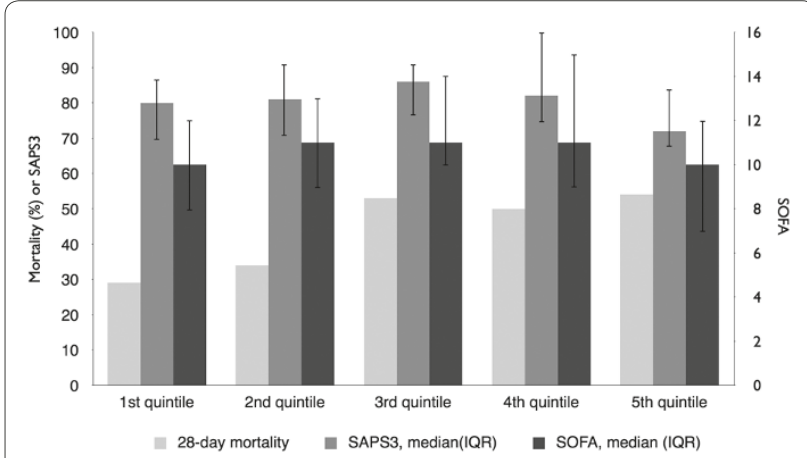

Figure 1. Trends in 28-day mortality rate, Simplified Acute Physiology Score 3 (SAPS 3) and Sequential Organ Failure Assessment (SOFA) score according to time to initiation of lowdose corticosteroid therapy after development of septic shock in quintiles $(P=0.0107, P=0.9953$ and $P=0.8437$, respectively, tests for trend). Error bars indicate the interquartile ranges (IQR) of SAPS 3 and SOFA scores.

differences occur or how many differences actually occur [2]. We examined the trends of severity of illness across 
the quintile of time to initiation of low-dose corticosteroid therapy with Jonckheere-Terpstra test [2]. As a result, severity of illness assessed by SAPS 3 and SOFA scores was not different across the quintiles of time to initiation of low-dose corticosteroid therapy $(P=0.9953$ and $P=0.8437$, respectively) (Figure 1). Therefore, the finding of increased mortality in patients receiving delayed initiation of low-dose corticosteroid therapy was not related to their increased severity of illness.

Abbreviations

SAPS, Simplified Acute Physiology Score; SOFA, Sequential Organ Failure Assessment.

\section{Competing interests}

The authors declare that they have no competing interests.

Published: 23 April 2012

\section{References}

1. Park HY, Suh GY, Song JU, Yoo H, Jo IJ, Shin TG, Lim SY, Woo S, Jeon K: Early initiation of low-dose corticosteroid therapy in the management of septic shock: a retrospective observational study. Critical Care 2012, 16:R3.

2. Bewick V, Cheek L, Ball J: Statistics review 10: further nonparametric methods. Crit Care 2004, 8:196-199.

doi:10.1186/cc11196

Cite this article as: Sprung $C L$, Batzofin BM: Early administration of corticosteroids and mortality. Critical Care 2012, 16:423. 